\title{
Robust Focus Measure for Low-Contrast Images ${ }^{1}$
}

\author{
Chun-Hung Shen and Homer H. Chen \\ Graduate Institute of Communication Engineering, National Taiwan University \\ Taipei, Taiwan, Republic of China \\ r93942021@ntu.edu.tw, homer@cc.ee.ntu.edu.tw
}

\begin{abstract}
The problem of auto-focusing has been studied for long, but most techniques found in literature do not always work well for low-contrast images. In this paper, a robust focus measure based on the energy of the image is proposed. It performs equally well on ordinary and low-contrast images. In addition, it is computationally efficient.
\end{abstract}

\section{INTRODUCTION}

Acquiring clear and well-focused images is an important task for most image-related applications such as medical imaging, remote monitoring, and photography. The problem of auto-focusing has been studied for long [1], [3]-[5], but most techniques found in the literature do not always work well for low-contrast images acquired under, for example, dim light condition.

A defocused image can be thought of as the image generated by convolving a focused image with a low-pass filter [1] whose bandwidth is proportional to the degree of focus. If the image is less focused, it loses more details. Most auto-focusing techniques are based on this basic property of image focusing. These techniques work by assigning more weight to the high frequency components of the image when evaluating the degree of focusing. For low-contrast images, there are fewer components in the high frequency range. Therefore, a technique based on the above basic property of image focusing would experience difficulty in focus detection for low-contrast images. The goal of our work described in this paper is to overcome this weakness of most auto-focusing techniques. The proposed robust algorithm is capable of handling noisy and low-contrast images.

The paper is organized as follows. Four different focus algorithms employing image variance, normalized variance [2], Sobel operators [3], and Laplacian mask [1] are reviewed in Section II. Then a robust focus algorithm based on the energy of the image is described in Section III. Finally, in Section IV these algorithms are compared and discussed.

\section{REVIEW}

To measure the degree of focus of an image, an index called "focus value" has been used. The focus value reaches a maximum for the best focused image and decreases as the image blurs. A good focus value should satisfy the following basic requirements. First, it should be content-independent,

\footnotetext{
1 This work was supported in part by the National Science Council of Taiwan under contract NSC94-2752-E-002-006-PAE.
}

which means it must not assume any particular structures in the image (e.g., isolated bright points). Second, it should be monotonic with respect to the image blurriness. The more blurred the image is, the less the focus value should be. Third, it should have good discrimination power; that is, it should give a more sharply response when the focus point is closer. The sharper the focus peak is, the easier it is to focus the image accurately. Finally, the focus value should be able to combat noisy and low-contrast imaging conditions.

One of the commonly-used focus measures is to compute the variance of image intensity [1], [2]. This method has been proved to be monotonic and equal to the sum of square of the AC coefficients of the image in discrete cosine transform (DCT) domain [7]. Another algorithm called "normalized variance" [2] normalizes the variance by the mean value of the image, and thereby compensates for the mean value of different images. These two variance-based methods are fast and robust. However, they have poor discrimination power.

In the "Tenengrad" method [3], [6], the horizontal and vertical Sobel operators are used to evaluate the strength of the horizontal and vertical gradients of the image. Then the sum of square of the gradients is defined as the focus value. Similarly, the method in [1] convolves an image with the Laplacian mask and defines the sum of square of the pixels in the resulting image as the focus value. In contrast to the variance-based methods, these two methods in general have good discrimination power. However, they (especially the Laplacian-based method) are more sensitive to noise than the variance-based methods.

All the techniques discussed so far perform the focus measure in the image domain. Transform-domain techniques are discussed next.

\section{PROPOSED METHOD}

The main idea behind our algorithm is simple. Let $E_{A C}$ be the energy of the AC part of the DCT image. In other words, $E_{A C}$ is the sum of square of the AC coefficients. Similarly, let $E_{D C}$ be the square of the DC coefficient. As opposed to using the AC energy directly as the focus value [7], we calculate the ratio between $E_{A C}$ and $E_{D C}$ and define it as the focus value. By dividing the energy of the high-frequency band by that of the low-frequency band, our new formula for measuring the focus value gives rise to more homologous results than the "normalized variance" method. As a result, it works equally well for ordinary images as well as low-contrast images. In addition, the focus value is calculated block-by-block. (The 
block size is $4 \times 4$ in our current implementation.) In contrast to the approach where the variance is calculated on a global basis for the entire image [2], the local variances computed this way in our algorithm carry more information about the image discontinuities. Therefore, it responds well to the high frequency components and has more discrimination power than other variance-based methods.

\section{Simulation Results}

In all experiments described in this section, the sizes of test images are all $64 \times 64$ pixels. Two of the test images are shown in Fig. 1.

In the first experiment, the test image shown in Fig.1(a) is used. The resulting focus value is plotted against 14 different camera's lens positions, from near to far, in Fig. 2. The 8th lens position is the one with the best focus. To fit different focus values to the same range on the vertical axis and to get a fair comparison with the results by Krotkov [4], each curve in the plot is scaled to the $(0,1)$ range. From Fig. 2, we can see that the proposed algorithm performs best among these algorithms. To see how well the algorithm can combat noises, additive Gaussian noise with standard deviation 5 is introduced to the test image. The results are shown in Fig. 3. As we can see, the proposed algorithm has more robust performance than other methods.

In the last experiment, the low-contrast image shown in Fig. 1(b) acquired at four different lens positions from near to far is used for testing. The 4th lens position is the one with the best focus. The results shown in Fig. 4 clearly indicate that, while others fail, the proposed algorithm is able to determine correctly the focus position. Table 1 lists the average computing time taken by different algorithms. As we can see, the proposed method is almost 6.3 times faster than the Laplacian-based method.

\section{CONCLUSION}

In this paper, we have described a robust method for focus measure of low-contrast images. The method is simple and effective. It provides the same discrimination power as that of the Laplacian-based method, which is often regarded as the best method for ordinary images. But our method is more robust and 6.3 times faster. In addition, our method works well for ordinary images as well as low-contrast images.

\section{REFERENCE}

[1] M. Subbarao, T. Choi, and A. Nikzad, "Focusing techniques," J. Opt. Eng., vol. 32, pp. 2824-2836, 1993.

[2] T. T. E. Yeo, S. H. Ong, Jayasooriah, R. Sinniah, "Autofocusing for tissue microscopy", Image and Vision Computing, vol. 11, no. 10, pp. 629-639, December 1993.

[3] J. M. Tenenbaum, "Accommodation in computer vision," Ph.D. dissertation, Stanford Univ., Stanford, CA, 1970.

[4] E. Krotkov, "Focusing," Int. J. Computer Vision, vol. 1, pp. 223-237, Oct. 1987.

[5] R. A. Jarvis, "Focus Optimisation Criteria for Computer Image Processing," Microscope, vol. 24, no. 2, pp. 163-180, 1976.

[6] J. F. Schlag, A. C. Sanderson, C. P. Neumann, and F. C. Wimberly, "Implementation of automatic focusing algorithms for a computer vision system with camera control," Carnegie Mellon Univ., Pittsburgh, PA, CMU-RI-TR-83-14, Aug. 1983.

[7] J. Baina, and J. Dublet, "Automatic Focus and Iris Control for Video Cameras", IEEE Fifth International Conference on Image Processing and its Applications, pp.232-235, 4-6 July 1995.

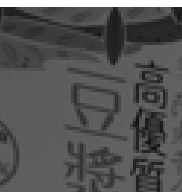

(a)

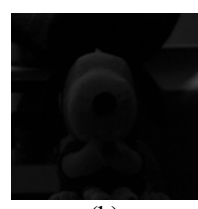

(b)
Fig. 1. a) A normal test image. b) A low-contrast image.

TABLE I

AVERAGE COMPUTING TIME

\begin{tabular}{ll}
\hline \hline Algorithm & Average Time $(\mu \mathrm{s})$ \\
\hline (1) proposed & 59.68 \\
(2) Tenengrad & 352.65 \\
(3) Laplacian-based & 374.20 \\
(4) variance & 36.97 \\
(5) normalized variance & 37.12
\end{tabular}

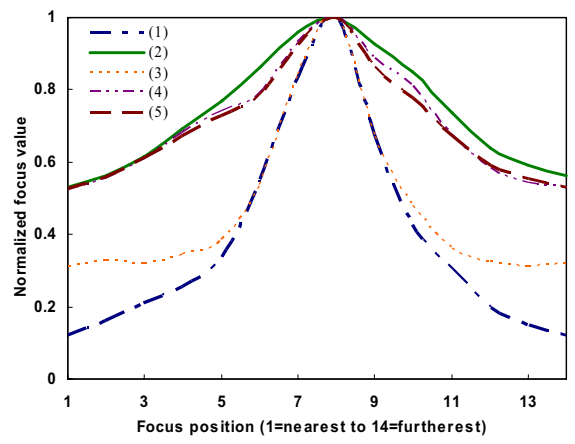

Fig. 2. Focus values obtained by different methods. 1) Proposed, 2) Tenengard, 3) Laplacian-based, 4) variance, and 5) normalized variance.

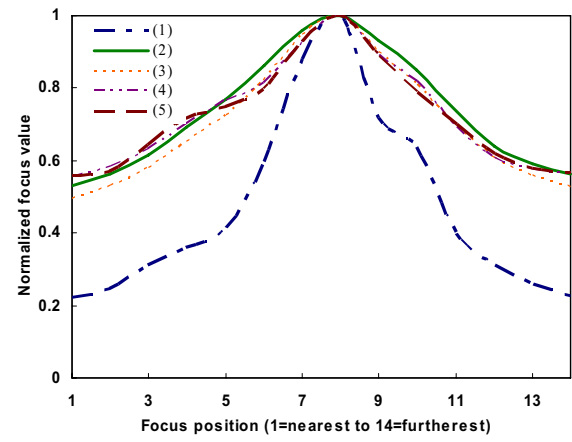

Fig. 3. Focus values obtained by different methods for noisy image. 1) Proposed, 2) Tenengard, 3) Laplacian-based, 4) variance, and 5) normalized variance.

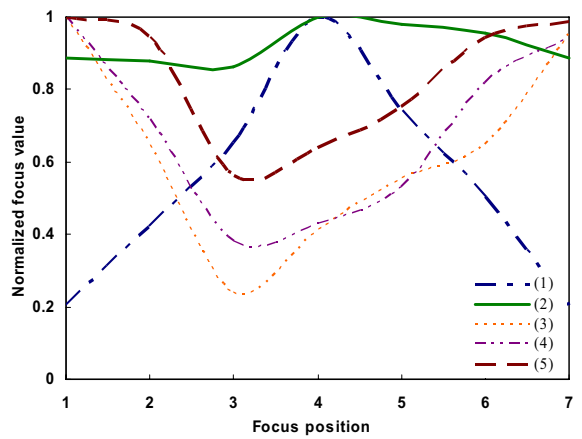

Fig. 4. Focus values obtained by different methods for low-contract image. 1) Proposed, 2) Tenengard, 3) Laplacian-based, 4) variance, and 5) normalized variance. 